\title{
La relación entre la política de defensa y su dimensión internacional. El caso argentino (2011-2015)
}

\section{The relationship between defense policy and its international dimension. The Argentine case (2011-2015)}

\section{INTRODUCCIÓN}

El presente artículo sostiene que la política exterior precede analíticamente a la política de defensa y se encuentra fuertemente imbricada con ésta. Si esto fuera así, las orientaciones de la política exterior deberían reflejarse en los lineamientos de la política de defensa. Esta afirmación no es nueva, en tanto que es muy conocida la distinción entre la maniobra militar y la maniobra diplomática. ¿Pero de qué manera la política exterior, a su vez, se relaciona y contribuye con la política de defensa? Además, ¿qué sucede en tiempos de paz y en un contexto de incertidumbre cuándo el enemigo no es conocido? Claramente, la política exterior se convierte en una herramienta fundamental para mitigar los riesgos que se adoptan en ese ambiente internacional. El diseño del instrumento militar no es un proceso sencillo, requiere tiempo y es inelástico a los cambios estratégicos que puedan producirse en el escenario internacional y regional en el corto plazo. El planeamiento por capacidades acentúa estas incertidumbres porque pone el énfasis en el cómo enfrentar diferentes tipos de amenazas y no en el quién, que es desconocido. Los supuestos que se adoptan, entonces, suponen riesgos que deben ser compensados por la política exterior.

A los efectos de estudiar dicha relación se analiza la política de defensa argentina, puntualmente, sus lineamientos estratégicos y las característi-

Sergio Gabriel Eissa - Licenciado en Ciencia Política (UBA); Magíster en Ciencias Sociales mención en Relaciones Internacionales dela Facultad Latinoamericanade Ciencias Sociales (FLACSO) Sede Argentina; y Doctor en Ciencia Política dela UniversidadNacionalde San Martín (UNSAM). 
cas del planeamiento por capacidades, y, posteriormente, la política exterior en su dimensión de defensa entre los años 2011 y 2015. En primer lugar, el estudio del vínculo analítico entre ambas es relevante porque mientras entre los años 2009 y 2011 se desarrolló el Primer Ciclo de Planeamiento; la implementación de su principal producto, el Plan de Capacidades Militares (PLANCAMIL 2011) se efectuó - valga la redundancia - entre el 2012 y el 2015 y que coincidió, además, con la puesta en marcha del Segundo Ciclo de Planeamiento para la Defensa Nacional. En segundo lugar, el caso argentino es teóricamente importante porque la transición a la democracia se produjo por colapso en el año 1983, es decir, no hubo pacto ni acuerdo con la última dictadura militar (1976-1983) y porque se alcanzó el control civil de las Fuerzas Armadas tempranamente en 1990. En tercer lugar, y pese a lo expuesto precedentemente, las autoridades políticas se desentendieron de la política de defensa entre 1990 y 2005, al punto tal que la Ley de Defensa de 1988 sólo fue implementada a partir del año 2006. Su implementación parcial supuso también completar el entramado normativo que posibilitó la puesta en marcha del Primer Ciclo de la Defensa Nacional, cuyas enseñanzas alimentaron el Segundo Ciclo que analizaremos en este trabajo (Eissa 2015).

En el presente artículo se sostendrá que la postura estratégica defensiva y el planeamiento por capacidades adoptados, que implica la aceptación de riesgos políticos y estratégicos, requieren una política exterior particularmente activa y contribuyente a la defensa nacional.

Para ello, y en primer lugar, se recurre al marco analítico denominado Glass Onion (Eissa 2015) que recurre eclécticamente a conceptos de las políticas públicas y las relaciones internacionales. En segundo lugar, y en lo que respecto a la metodologia, se analizarán las manifestaciones públicas de los actores políticos y sociales a través de distintos medios y fuentes secundarias (producción académica referida a esta temática) y los documentos elaborados por el nivel estratégico nacional (Poder Ejecutivo, Ministerio de Defensa y la Cancillería) y el nivel estratégico militar (Fuerzas Armadas). La triangulación (Mendicoa 2003) permite contar con otros caminos para tener una comprensión e interpretación más acabada del fenómeno En cuanto a los documentos, Taylor (1984: 149-150) sostiene que "el investigador cualitativo analiza los documentos públicos y oficiales para adquirir conocimiento sobre las personas que los redactan [...], lo cual abre muchas nuevas fuentes de comprensión”. Entre algunos de los documentos a relevar, se encuentran el Libro Blanco de la Defensa 2010 y 2015; leyes como la Ley de Defensa Nacional, la Ley de Seguridad Interior, la Ley de Reestructuración de las Fuerzas Armadas y la Ley de Inteligencia; decretos del Poder Ejecutivo; documentos específicos del ni- 
vel estratégico nacional y del nivel estratégico militar. No es propósito en este artículo la dinámica de los actores al interior del Ministerio de Defensa y en relación con el Estado Mayor Conjunto y las Fuerzas Armadas.

A los efectos de avanzar en el análisis, el artículo se estructura en cuatro partes. En la primera se define la relación analítica entre política exterior y política de defensa. En el segundo apartado, se desarrollan los lineamientos estratégicos de la política de defensa argentina. Acto seguido se analiza el planeamiento por capacidades militares, diferenciándolo, por ejemplo, del planeamiento por hipótesis de conflicto. A continuación, se estudia la política exterior, especialmente en su relación con la política de defensa, en el período señalado. Finalmente, se exponen algunas breves conclusiones sobre la relevancia analítica de esta relación y su materialización durante los años de estudio propuestos.

\section{CONSIDERACIONES ANALÍTICAS}

En primer lugar, se entiende la política exterior como "la acción política gubernamental que abarca tres dimensiones analíticamente separables político-diplomática, militar-estratégica y económica - y que se proyecta al ámbito externo frente a una amplia gama de actores e instituciones gubernamentales y no gubernamentales, tanto en el plano bilateral como multilateral” (Russell 1990, 255; Colacrai 2004, 2). En este sentido, se considera a la política de defensa del país como íntimamente asociada con la política exterior y ésta precede analíticamente a la primera. Por lo tanto, no se puede dar cuenta de una sin explicitar los principales lineamientos de la otra (Eissa 2015).

En segundo lugar, y siguiendo a Jorge Battaglino (2010, 242), la política de defensa puede ser definida como "el conjunto de acciones que adopta un Estado para garantizar su supervivencia frente a riesgos y amenazas" externas de origen estatal. Asimismo, se argumenta que la política de defensa tiene al menos tres dimensiones: "política de defensa en sentido estricto" (en adelante, la dimensión estratégica); "política militar”; y la "dimensión internacional de la política de defensa". ${ }^{1}$ Mientras la política militar expresa la necesidad de ejercer el gobierno político sobre tal organización (Battaglino 2010), la política de defensa en sentido estricto consistiría de:

las acciones, actitudes y medidas institucionales de carácter estratégico, decididas e implementadas por el gobierno nacional y destinadas a prevenir o enfrentar distintos tipos de situaciones de riesgo, conflictos o amenazas, potenciales o efectivas, que provengan de agresiones estatales de origen externo y que hagan peligrar la in- 
tegridad territorial y la capacidad de autodeterminación del Estado y que requieran el empleo de las Fuerzas Armadas, en forma disuasiva o efectiva. ${ }^{2}$

Por último, la dimensión internacional de la política de defensa se constituye en punto de contacto entre la política de defensa (en términos generales) y la política exterior (Russell 1990); esto es, las acciones que se realizan desde la política de defensa como contribución a la política exterior de un país.

En síntesis, si el vínculo analítico planteado es correcto, debería haber una correspondencia entre política exterior y política de defensa. Es decir, cabe pensar que el proceso de integración regional y la decisión de eliminar las hipótesis de conflicto que planteaban una solución militar con los países vecinos tuvieron que haber impactado en los lineamientos de la política de defensa argentina. Pero, por otro lado, se sostiene que dicha relación analítica es de ida y vuelta, en tanto que la política de defensa requiere de la política exterior para que la misma sea factible en el mediano plazo. En efecto, si la supresión de las hipótesis de conflicto con los países de la región y el establecimiento de hipótesis de confluencia impuso la necesidad de adoptar la metodología de planeamiento por capacidades militares; este tipo de planeamiento requiere el acompañamiento de la política exterior, en especial, la dimensión internacional de la política de defensa para paliar los potenciales riesgos asociados a esta metodología.

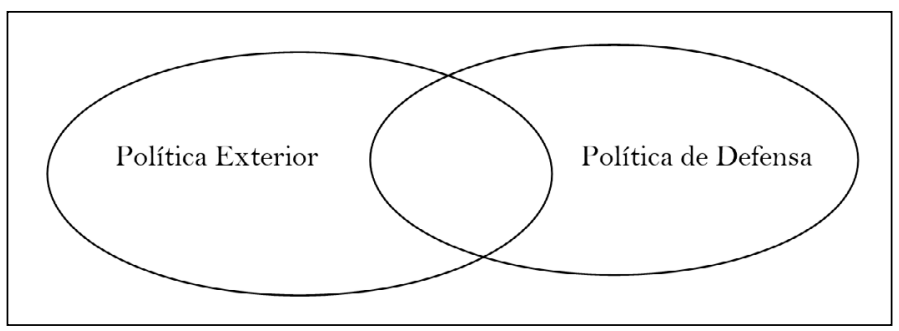

Gráfico 1 - Política Exterior y Política de Defensa. Fuente: elaboración propia.

\section{CONSIDERACIONES SOBRE LOS LINEAMIENTOS POLÍTICO-ESTRATÉGICOS DEL SISTEMA DE DEFENSA ARGENTINO}

A partir de 1985, diversos actores políticos y sociales, radicales, ${ }^{3}$ peronistas $^{4}$ e independientes comenzaron a trabajar en un proyecto de ley de defensa. Como telón de fondo de dicha iniciativa, se encontraba la urgencia 
de propiciar una institucionalización fuerte en la materia, que significara cerrar cualquier puerta a una nueva intervención militar. Pero asimismo, se consideraba necesario propiciar un cambio doctrinario que extirpara del sector castrense la Doctrina de Seguridad Nacional, sobre cuya base se había justificado la intervención militar en asuntos de seguridad interior y la ejecución de una brutal represión que condujo a masivas violaciones de los derechos humanos. Tal proyecto de ley debía entonces consensuar una definición de defensa nacional que sentara las bases para la construcción de una política de defensa autónoma. Independientemente de las motivaciones de los actores involucrados, ${ }^{5}$ la convergencia de sus intereses y sistemas de creencias permitieron alcanzar en 1988 lo que Marcelo Saín (2010) ha denominado el "consenso básico" (ver Gráfico 2).

La fortaleza de esa problematización de la defensa nacional permitió que el "consenso básico" cristalizara en tres leyes esenciales a lo largo de tres gobiernos y de trece años. Nos referimos así a la Ley $\mathrm{N}^{\circ} 23.554$ de Defensa Nacional, aprobada en el año 1988; la Ley Nº 24.059 de Seguridad Interior, de 1992; y la Ley $\mathrm{N}^{\circ} 25.520$ de Inteligencia Nacional, sancionada en el 2001.

Estas normas conforman así un constructo sistémico - en tanto que para su comprensión deberían ser leídas de manera conjunta - , articulado alrededor de tres principios básicos que consideramos como liminares. Dichos principios son: ${ }^{6}$

a) La supresión de las hipótesis de conflicto con los países vecinos que requieran la utilización de las Fuerzas Armadas;

b)La separación orgánica y funcional entre defensa nacional y seguridad interior; y

c) El gobierno civil de la política de defensa.

Dicho "consenso básico", que se materializó en las leyes citadas ut supra, fue fortaleciéndose durante los gobiernos de Néstor Kirchner (20032007) y Cristina Fernández de Kirchner (2007-2015), quienes aprobaron diversas normas de distinta jerarquía jurídica que hicieron operativas las leyes ya sancionadas y que completaron el entramado jurídico de la defensa nacional. En este marco, se consolidó una arquitectura institucional que posibilitó, entre otras cosas, la efectiva conducción política de las Fuerzas Armadas. Por lo tanto, puede sostenerse que la orientación de la política de defensa argentina se ha construido a partir de esos pilares y, en este proceso, cada gobierno ha agregado un elemento más en su consolidación, lo cual da cuenta del amplio arraigo del "consenso básico". 


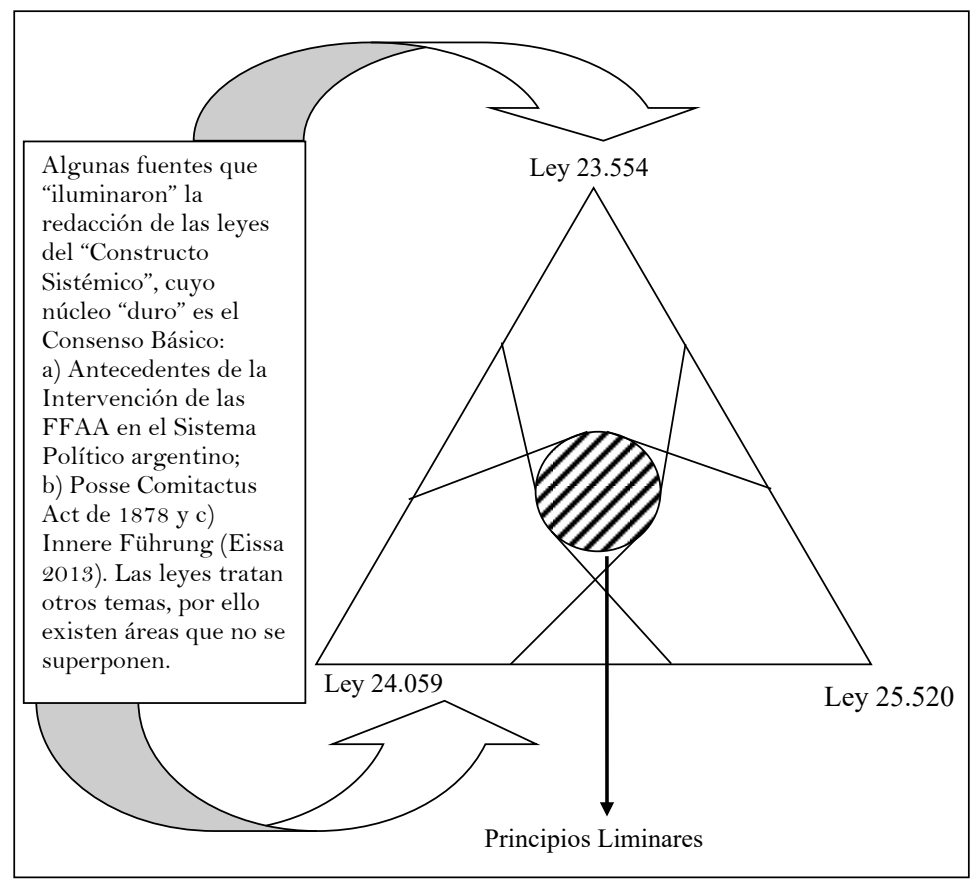

Gráfico 2 - Consenso Básico.

Fuente: elaboración propia.

¿Qué lineamientos se desprenden de estos principios liminares en relación al planeamiento por capacidades?

En primer lugar, la Ley de Defensa Nacional $\mathrm{N}^{\mathrm{o}} 23.554$ y su Decreto Reglamentario $\mathrm{N}^{\mathrm{o}}$ 727/2006 consideran que las Fuerzas Armadas, instrumento militar de la defensa nacional, serán para "enfrentar las agresiones de origen externo", "perpetradas por fuerzas armadas pertenecientes a otro/s Estado/s". Asimismo, agrega que "se entenderá como agresión de origen externo el uso de la fuerza armada por un Estado contra la soberanía, la integridad territorial o la independencia política de nuestro país, o en cualquier otra forma que sea incompatible con la Carta de las Naciones Unidas".

En segundo lugar, nuestro país adopta una estrategia disuasiva y de la legítima defensa, de acuerdo al artículo 51 de la Carta de las Naciones Unidas. En virtud de lo expuesto, nuestro país adopta un posicionamiento y actitud estratégica defensiva, basada en el concepto de legítima defensa, como "criterio esencial y ordenador sobre la cual se estructura todo el sistema de defensa del Estado argentino”. 
En tercer lugar, el Instrumento Militar argentino debe estructurarse doctrinaria, orgánica y funcionalmente sobre la base de su misión principal, "cual es la de conjurar y repeler toda agresión externa perpetrada por Fuerzas Armadas de otro Estado". Por lo tanto, "la misión principal de las Fuerzas Armadas debe ser [...] el principal criterio ordenador de todo su diseño de fuerza, en tanto que toda misión subsidiaria del instrumento militar no deberá afectar las capacidades requeridas para el cumplimiento de aquella misión primaria y esencial”.

Finalmente, se considera que el Instrumento Militar sólo resultará apto: si se lo concibe, planifica y ejecuta como instrumento integrado, razón por la cual la acción militar deberá entenderse necesaria e ineludiblemente como acción integrada, esto es, como acción conjunta, incluso en aquellos casos en los que por el ámbito en que ésta se desarrolle y/o por las características propias de la operación en cuestión, la misma deba ser ejecutada por una fuerza específica en forma exclusiva.

Esta concepción se integra con la disposición que establece que "el Comando Operacional será la instancia responsable de la ejecución del adiestramiento conjunto, del control de las ejercitaciones conjuntas, de la elaboración del planeamiento estratégico operacional y de su correspondiente ejecución y de las operaciones militares” y que el Ejército, la Armada y la Fuerza Aérea sólo alistan, adiestran y sostienen los medios puestos a su disposición.

\section{CONSIDERACIONES SOBRE EL PLANEAMIENTO BASADO EN CAPACIDADES}

En sintonía con las decisiones adoptadas en materia de política exterior con los países de la región y con los lineamientos de la política de defensa, que establecieron su postura estratégica, cabe destacar que el diseño de las Fuerzas Armadas se realiza a través del método de planeamiento por capacidades en reemplazo del modelo basado en hipótesis de conflicto, tal como ha sido plasmado en los Decretos $\mathrm{N}^{\mathrm{o}}$ 1.691/2006 y No 1729/2007.

El empleo de este método se generalizó hacia fines de la Guerra Fría (1947-1991), como uno más entre muchas tendencias en el planeamiento, que trataron de innovar los sistemas para adaptarlos a "los nuevos tiempos" (Arteaga et al. 2007, 192-193). En tal sentido, se puede asegurar que la finalización del conflicto este-oeste marcó un punto de inflexión en los procedimientos tradicionales de planeamiento de la defensa. 
Mientras que durante la Guerra Fría, la base del planeamiento consistía en la evaluación de las amenazas militares externas y se traducía en un cálculo de balance de fuerzas (Arteaga Martín et al, 2007); contrariamente, en el actual escenario de incertidumbre, los planificadores de la defensa en lugar de señalar "quién”, han priorizado la identificación del "cómo", es decir, reconocer modos genéricos de agresión y establecer las capacidades necesarias para enfrentar tales tipos de amenazas.

Debe destacarse que la obtención de capacidades no es sinónimo de obtención de medios militares - por ejemplo, equipamiento y armas — dado que por capacidad se entiende la aptitud para lograr un efecto, es decir, "se trata de desarrollar determinadas aptitudes militares genéricas como la movilidad, la protección, la logística integrada, el mando y control y otras que se consideren necesarias para hacer frente a los retos operacionales del futuro" (Arteaga and Fojón Lagoa 2007, 204)”. Así, las capacidades no se diseñan pensando en un agresor concreto, sino en las aptitudes necesarias para combatir en el espectro más amplio posible de las operaciones militares. Este planeamiento evita que "los medios acaben determinando los fines” y que se perpetúen sistemas de armas y organizaciones que en algún momento se consideraron necesarios (Arteaga et al. 2007, 204, 209). Asimismo, mientras en el planeamiento tradicional se adecuaban las fuerzas a amenazas predeterminadas, a través de esta metodología se diseñan capacidades que permitan reaccionar frente a agresores militares que puedan surgir imprevistamente. En efecto, según Antonio Torres (2009, 58 y 63), el diseño del Instrumento Militar resultante es "mucho más dinámico, flexible e integral que el anterior (hipótesis de conflicto)".

Como puede observarse, el método de planeamiento por capacidades resulta idóneo para aquellos Estados cuya situación estratégica no observa una situación de conflicto bélico inminente ni en el mediano o largo plazo, o una potencial agresión, siendo adecuado para los países que se encuentran en una zona de paz. Por ello, el proceso de planeamiento por capacidades se inicia identificando escenarios donde se puede emplear al instrumento militar, los que pueden tomar la forma de "escenarios estratégicos" o "operativos”, que sirven para establecer las funciones que deben realizar las fuerzas armadas a mediano y largo plazo. Es decir, los escenarios deben reflejar el tipo de misión que el Nivel Estratégico Nacional establece para el empeñamiento del instrumento militar. Por tal motivo, Davis (2002) sugiere desarrollar junto a la elaboración de escenarios, un análisis de misiones, lo cual brinda mayor precisión para la definición de las capacidades a desarrollar.

Este método de planeamiento fue plasmado en el caso argentino a través del Decreto No 1729/2007 que aprobó el "Ciclo de Planeamiento de la 
Defensa Nacional”. Dicho proceso se inicia con el dictado de la Directiva de Política de Defensa Nacional (DPDN), suscripta por la presidenta de la Nación, a propuesta del Ministerio de Defensa, y concluye con la elaboración, también por parte del Nivel Estratégico Nacional, del Plan de Capacidades Militares (PLANCAMIL), documento que determina el modelo de evolución del Instrumento Militar, posible y necesario, para el mediano plazo.

La Directiva de Política de Defensa Nacional, que fue aprobada tardíamente por la presidenta Cristina Fernández de Kirchner a través del Decreto $\mathrm{N}^{\mathrm{o}}$ 1.714/2009, puso en marcha el Primer Ciclo de Planeamiento de la Defensa Nacional en el año 2009. Éste precisa cuestiones centrales relativas al posicionamiento y la actitud estratégica que adopta la República Argentina en materia de defensa, definiendo las misiones, los criterios de empleo, la estructura organizacional y las capacidades y equipamiento de las fuerzas armadas. Este documento, que inicia el Ciclo de Planeamiento de la Defensa Nacional, permite dar racionalidad a todo el proceso, en tanto los lineamientos, orientaciones y directrices de Nivel Estratégico Nacional para el empleo del instrumento militar allí planteadas deben guiar, articular, encuadrar y definir el diseño de las capacidades de las fuerzas armadas, es decir el Planeamiento Estratégico Militar, bajo la responsabilidad del Estado Mayor Conjunto de las Fuerzas Armadas. De tal modo se asegura que sean los "fines" los que determinen los "medios", y no inversamente. Así las capacidades del Instrumento Militar resultantes de este proceso de planeamiento y sus factores componentes (material, infraestructura, recursos humanos, información, logística, adiestramiento, doctrina y organización - MIRILADO) deben responder a tales criterios. De lo contrario, las capacidades resultantes serán insuficientes para cumplir el efecto deseado, perdiendo toda racionalidad el proceso.

Una vez aprobada la Directiva de Política de Defensa Nacional, el Ciclo de Planeamiento continúa con la aprobación por parte del Ministerio de Defensa, de la Directiva para la Elaboración del Planeamiento Estratégico Militar (DEPEM), elaborada por el Estado Mayor Conjunto de las Fuerzas Armadas. A partir de esta instancia, se inicia el planeamiento estratégico militar propiamente dicho, el cual se materializa a través de los siguientes documentos: 


\section{Cuadro 1}

Documentos y Responsables del Planeamiento de la Defensa

\begin{tabular}{|l|l|}
\hline Nombre del Documento & Responsable de la Elaboración \\
\hline $\begin{array}{l}\text { Apreciación y Resolución Estratégica } \\
\text { Militar }\end{array}$ & $\begin{array}{l}\text { Estado Mayor Conjunto de las Fuerzas } \\
\text { Armadas }\end{array}$ \\
\hline Directiva Estratégica Militar & $\begin{array}{l}\text { Estado Mayor Conjunto de las Fuerzas } \\
\text { Armadas }\end{array}$ \\
\hline $\begin{array}{l}\text { Planes Militares de Corto, Mediano y } \\
\text { Largo Plazo }\end{array}$ & $\begin{array}{l}\text { Estado Mayor Conjunto de las Fuerzas } \\
\text { Armadas }\end{array}$ \\
\hline $\begin{array}{l}\text { Proyecto de Capacidades Militares } \\
\text { (PROCAMIL) }\end{array}$ & $\begin{array}{l}\text { Estado Mayor Conjunto de las Fuerzas } \\
\text { Armadas }\end{array}$ \\
\hline Plan/es Estratégico/s Operacional/es & Comando/s Operacional/es \\
\hline Planes de Apoyo & $\begin{array}{l}\text { Ejército Argentino, Armada Argentina y Fuerza } \\
\text { Aérea Argentina }\end{array}$ \\
\hline
\end{tabular}

Fuente: elaboración propia.

Es posible señalar que la decisión de haber cambiado el método de planeamiento por hipótesis de conflicto a un planeamiento basado en capacidades supone un desafío y un salto cualitativo, pero también implica un peligro.

La metodología anterior era relativamente sencilla en un contexto de certidumbre, en tanto que el poder político había definido cuáles eran los "enemigos"; a partir de lo cual se diseñaba el Instrumento Militar para hacerles frente. En forma simplificada, el conocer cuántos barcos, tanques y aviones (y las características de éstos) que el oponente disponía permitía organizar y establecer cuáles medios debían tener las propias fuerzas armadas, por lo cual era muy probable, en aquellos países que recurrían a este modelo de planeamiento, impulsar carreras armamentistas. El nuevo método, contrariamente, parte de la apreciación de un escenario en el que la República Argentina no tiene hipótesis de conflicto que requieran el empleo del Instrumento Militar, por lo que no se puede identificar anticipadamente un enemigo (situación que se refuerza en el marco de la zona de paz sudamericana). De esta manera, el nuevo método de planeamiento pone el acento en el "cómo" y el "qué", y no en el "quién”. Es decir, se busca establecer que efectos militares debe ser capaz de producir el Instrumento Militar para cumplir con su misión principal y cómo debe producirlos.

Ahora bien, si el planeamiento de capacidades parte de la premisa de la incertidumbre, se corre el peligro de diseñar un instrumento militar para todo "tipo" de escenarios genéricos. En el caso argentino, el peligro sería diseñar un Instrumento Militar para todo tipo de Amenaza Estatal Militar 
Externa (AEME). La pregunta que entonces surge es ¿tienen el mismo grado de probabilidad de ocurrencia para Argentina un ataque termonuclear que un ataque de un país chico? Otorgarle la misma probabilidad de ocurrencia implicaría diseñar un Instrumento Militar que posea todas las capacidades necesarias - aunque sea en su mínima expresión - para hacer frente a todas esas AEME. En otras palabras, implicaría diseñar un Sistema de Defensa Nacional que sería imposible de costear. Justamente, el propósito fundamental de esta metodología de planeamiento es "invertir los recursos escasos en las capacidades militares que mayor ventaja comparativa proporcionen" (Arteaga et al. 2007, 197). Por tal razón, esta metodología debe combinar la incertidumbre estratégica con la realidad presupuestaria.

En consecuencia, resulta relevante introducir como variable el grado de probabilidad de ocurrencia de determinado tipo de AEME. Al hacer esto se está aceptando un riesgo: éste puede ser definido como la probabilidad de que una agresión provoque daños significativos sobre los objetivos de valor estratégicos de la nación. Este riesgo puede ser, analíticamente, dividido en tres componentes:

- Riesgo político: se relaciona con las definiciones políticas en materia estratégica que los decisores políticos han adoptado. Esta es una definición política y, en el caso argentino, la apreciación del escenario regional desde la década del '80 es la progresiva disminución de la probabilidad de ocurrencia de un conflicto interestatal. En tal sentido, Argentina renunció unilateralmente a tener hipótesis de conflicto - no confundir con el método de planeamiento - con los países de la región, lo cual fue reforzado con acciones de política exterior tales como el Mercosur, el Tratado de Límites de 1985, el Tratado de Demarcación de 1998, entre otras medidas.

- Riesgo de diseño estratégico: es inherente a todo método de planeamiento bajo incertidumbre, en tanto se desconoce cuál de las formas genéricas de agresión - a partir de las cuales se planifica - es la que podría materializarse como agresión estatal militar externa. Este riesgo sólo podrá ser determinado con la aparición del conflicto concreto, el cual podrá despejar la incertidumbre respecto de la suficiencia de la cantidad de cada capacidad. A los efectos de planeamiento se construyen analíticamente tipos ideales de AEME y se planifica en función de aquellas que tienen mayor probabilidad de ocurrencia. En este sentido, se evita que el diseño del Instrumento Militar devenga en un sobredimensionamiento del mismo. 
- Riesgo de desarrollo: el proceso de adquisición de capacidades, a partir de un mínimo sistémico, conlleva necesariamente un riesgo, el cual irá disminuyendo en la medida que el proceso de transformación y de incorporación de las capacidades necesarias del Instrumento Militar para el mediano y largo plazo se vaya completando a lo largo del tiempo.

En función de lo expuesto, el concepto de riesgo está sujeto al cálculo probabilístico. Por tal motivo, dicho concepto requiere para su adecuada evaluación de un conocimiento exhaustivo de una variada gama de factores, que en el ambiente incierto propio de la estrategia militar no siempre está disponible. Por otro lado, los dos primeros tipos de riesgos pueden ser considerados como constantes en el mediano plazo a partir de la apreciación del escenario que se haya realizado. Mientras que el tercer tipo de riesgo es variable y tiende a disminuir en la medida en que las previsiones del planeamiento sean implementadas. Si se producen desvíos en la implementación por un aumento o disminución de los recursos aplicados al diseño del Instrumento Militar, el riesgo de diseño disminuirá o aumentará respectivamente.

El Ciclo de Planeamiento de la Defensa Nacional culmina - como se señaló - con un Plan de Capacidades Militares (PLANCAMIL), elaborado también por el Nivel Estratégico Nacional, es decir, el poder político, que materializa el Instrumento Militar necesario y posible porque es el que permite cumplir con la misión principal con un horizonte de recursos políticamente aceptable.

Finalmente, esta metodología de planeamiento se entronca, como se dijo con la política exterior, permitiendo eliminar los dilemas de seguridad entre los países de la misma región, desactivando así también el desarrollo de carreras armamentistas.

Cómo se puede observar en el Gráfico 3, la política exterior es la que compensa el riesgo asociado al planeamiento por capacidades (en el gráfico corresponde al área determinada debajo de la curva). Dicho de otra manera, la maniobra diplomática acompaña a la maniobra militar, pero en tiempos de paz y de transformación del Instrumento Militar, la política exterior debe minimizar el riesgo inherente a la postura estratégica elegida y a la metodología de planeamiento adoptada: política exterior, postura estratégica, política de defensa, método de planeamiento y política exterior, nuevamente, conforman una cadena de valor que contribuye a la seguridad estratégica. Cualquier ruptura en uno de los eslabones pone en riesgo no solo el desarrollo del Instrumento Militar, sino la seguridad del Estado. 
Dado que la implementación del PLANCAMIL debió ser modificad, en cuanto a los plazos previstos, por la falta de asignación presupuestaria, el riesgo de desarrollo se incrementó, por lo cual, la política exterior debió haber sido activa entre 2011 y 2015 para compensar el mismo. No se está sosteniendo que la política exterior suplante totalmente a la política de defensa, pero es imposible considerar una sin tener en cuenta la otra.

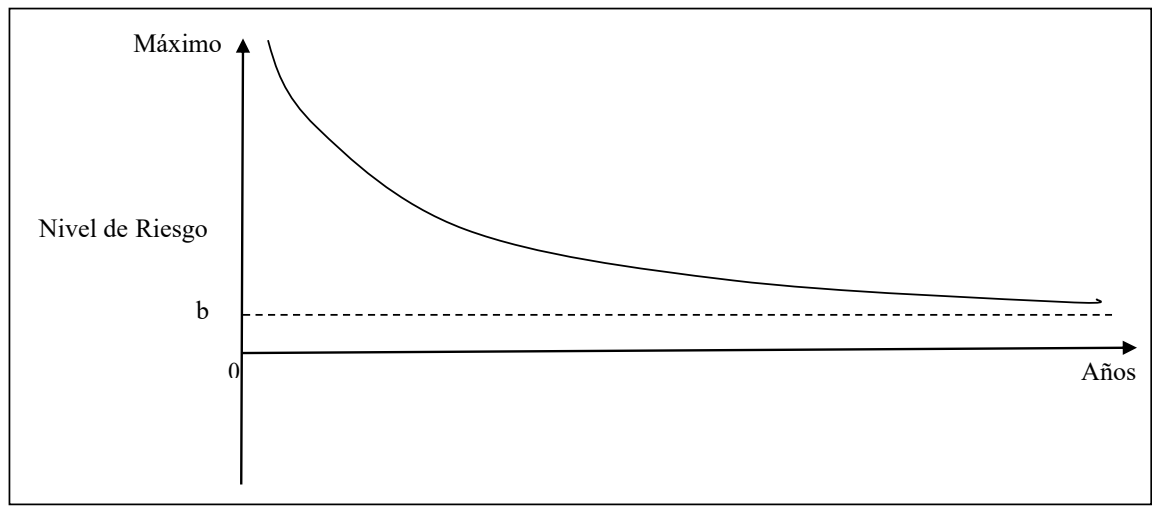

Gráfico 3: Riesgo, Política exterior y defensa.

Fuente: elaboración propia.

\section{POLÍTICA EXTERIOR ARGENTINA (2011-2015)}

\section{a) Algunos antecedentes}

Una de las constantes de la política exterior argentina es la integración con América Latina. Durante el primer gobierno democrático, encabezado por Raúl Alfonsín (1983-1989), se avanzó aún más de lo que se había hecho hasta entonces. A los efectos de este trabajo, se quiere destacar la firma del Programa de Integración y Cooperación Económica (PICE) con Brasil, al que luego se incorporó Uruguay; y el referéndum y posterior aprobación del Tratado de Paz y Amistad con la República de Chile en 1984, que solucionó la delimitación de la frontera en el Canal del Beagle y el Estrecho de Magallanes. Estos acuerdos significaron el abandono de las viejas hipótesis de conflicto, inspiradas en pasadas concepciones geoestratégicas; el punto inicial para la construcción de un mercado común (Sempé 1996); y el impulso para fortalecer la democracia, distendiendo dos de las fronteras más importantes del país (Fontana 2006). 
Estas acciones fueron continuadas por el presidente Carlos Menem (1989-1999). Durante su presidencia, se mantuvo la orientación de política exterior respecto a Brasil y Chile, se profundizó la distensión y se avanzó fuertemente en el proceso de integración con ambos países. En primer lugar, el 26 de marzo de 1991 se puso en marcha el Mercado Común del Sur (MERCOSUR), integrado por Argentina, Brasil, Paraguay y Uruguay, que vino a dar continuidad al ya mencionado PICE. Asimismo, se continuó avanzando en materia de cooperación nuclear con Brasil: la Declaración Conjunta sobre Política Nuclear de 1985 fue seguida por la Declaración sobre Política Común de Salvaguardas Nucleares en 1990, por la creación de la Agencia Brasileño-Argentina de Contabilidad y Control de Materiales Nucleares (ABACC) y un Sistema Común de Contabilidad y Control (SCCC) en 1991, y el Acuerdo de Salvaguardas entre el Organismo Internacional de Energía A tómica (OIEA) y los gobiernos de ambos países en 1991. De igual forma, Argentina y Brasil aprobaron el Tratado para la Proscripción de Armas Nucleares en América Latina y el Caribe (Tratado de Tlatelolco) en 1993 y el Tratado sobre la No Proliferación de las Armas Nucleares (TNP) en 1994. Finalmente, y casi simultáneamente, ambos países pusieron, formalmente, fin a sus respectivas hipótesis de conflicto en 1996 (Fraga 1998).

Los acuerdos de cooperación mencionados tuvieron también su correlato en el ámbito de la defensa nacional; Argentina y Brasil suscribieron un conjunto de mecanismos a los efectos de fortalecer las relaciones bilaterales en esta área, a saber:

- El Mecanismo Permanente de Consulta y Coordinación, conocido como "Mecanismo de Itaipava" ó 2 + 2: reunió a los Ministros de Defensa y de Relaciones Exteriores de ambos países desde 1997.

- El Mecanismo Permanente de Análisis Estratégico (MAE) que realiza anualmente el seguimiento e implementación de las decisiones adoptadas en el Mecanismo de Consulta y Coordinación y es preparatorio de cada reunión del $2+2$.

Asimismo, en el marco de la Reunión del Mecanismo de Consulta y Coordinación del 28 de abril de 2000 se creó el Grupo de Trabajo Bilateral de Defensa (GTBD), cuya Primera Reunión tuvo lugar en Brasilia, 26 de octubre de 2001.

En cuanto a Chile, luego de un largo proceso iniciado en 1992, el 29 de diciembre de 1998 el Congreso argentino aprobó el tratado que puso fin a la demarcación de una veintena de puntos que aún quedaban sin demarcar en la frontera con ese país (Eissa, 2005); entre ellos, los más importan- 
tes eran Laguna del Desierto, resuelto a través de un arbitraje latinoamericano - de acuerdo a lo dispuesto en el Tratado de 1984 - y Hielos Continentales, a través de un acuerdo entre las partes. Estos pasos fueron acompañados por un importante acercamiento entre los sistemas de defensa de ambos países. A fines de 1995, se estableció el Comité Permanente de Seguridad entre Argentina y Chile (COMPERSEG).

Durante la presidencia de Néstor Kirchner (2003-2007) fue relanzada la relación con Brasil en materia de defensa, que se encontraba en un impasse producto de las orientaciones de la política exterior argentina en la década de 1990. El 30 de noviembre de 2005, con motivo del vigésimo aniversario del encuentro entre los ex presidentes Alfonsín y Sarney, que constituyó el marco inicial del programa de integración entre Argentina y Brasil y, posteriormente, la base del MERCOSUR, los Presidentes Kirchner y Lula se reunieron en Iguazú para conmemorar la firma de la Declaración de Iguazú y la Declaración Conjunta sobre Política Nuclear de 1985. Durante la ocasión, los Cancilleres de ambos países firmaron una serie de acuerdos de cooperación en diferentes áreas, entre los que se incluyó un Acuerdo Marco en materia de defensa y un Protocolo Complementario al Acuerdo de Cooperación Científica y Tecnológica (firmado por ambos países en 1980). Dicho Acuerdo Marco permitió profundizar las acciones cooperativas en dicha área y revisar los mecanismos de diálogo existentes, previendo la creación de un nuevo Grupo de Trabajo Conjunto entre ambos Ministerios.

A la vez, la cooperación con la República de Chile fue sumamente importante, no solo por las reuniones del COMPERSEG, sino también porque, bajo la supervisión de CEPAL (Comisión Económica para América Latina y el Caribe, de la ONU), se acordó una metodología estandarizada común para la medición de los gastos en defensa. Si bien estos avances dieron cuenta de una cooperación sin precedentes, cabe destacar que el logro más significativo en materia de defensa con la República de Chile fue la constitución de la Fuerza de Paz Combinada Cruz del Sur.

En efecto, en octubre de 2003, en ocasión de una de las reuniones del COMPERSEG, Chile presentó una propuesta denominada "Medidas para implementar el entrenamiento de fuerzas destinadas a cumplir operaciones de mantenimiento de la paz y participación combinada en futuras operaciones de paz”, que fue analizada por el Ministerio de Defensa argentino, el Estado Mayor Conjunto de las FF.AA. y la Cancillería. Como resultado, el 29 de agosto de 2005, el Ministro de Defensa de Argentina y el Ministro de Defensa de Chile firmaron un Protocolo de Entendimiento en el cual se acordó impulsar un salto cualitativo en la relación de cooperación binacional en el ámbito de la defensa y seguridad desarrollando una Fuerza 
de Paz combinada binacional en el corto plazo. Para ello, se efectuó una estimación de costos y se convino un calendario de actividades para la conformación de la Fuerza de Paz Combinada "Cruz del Sur", comenzando en 2006 con la creación de Estado Mayor binacional y culminando en 2008 con la formación de la Fuerza de Paz y su puesta a disposición de la ONU.

Finalmente, no se puede dejar de señalar la participación argentina en la operación de paz en la República de Haití (MINUSTAH), en el que participaron también con efectivos Brasil y Chile, entre otros países de América Latina.

En síntesis, la política exterior no sólo contribuyó a distender las tradicionales tensiones con Brasil y Chile, asistiendo de esa manera a la consolidación de la democracia y a la integración regional, sino también, como sostiene David Pion-Berlín (2001), a coadyuvar a la subordinación de las Fuerzas Armadas al poder político. Pero asimismo, estas decisiones supusieron una definición política, por parte de los actores políticos argentinos, que se ha sostenido desde el retorno a la democracia y, como tal, ha alumbrado a la política de defensa: la supresión de las hipótesis de conflicto con Chile y Brasil.

\section{b) La segunda presidencia de Cristina Kirchner (2011-2015)}

Durante la segunda presidencia de Cristina Kirchner se continuó fortaleciendo la relación internacional de Argentina en materia de defensa con los países de la región, a partir de una priorización geográfica de acuerdo a "un esquema de círculos concéntricos", abarcando el primer círculo a América del Sur (SSAID 2013). En este sentido cabe destacar la participación activa en la creación de la Unión Suramericana de Naciones (UNASUR) a través del Tratado de Brasilia en el 2008. La creación del Consejo de Defensa Suramericano (CDS), aprobada en Costa do Sauípe, Brasil, en ocasión de la Cumbre Extraordinaria de la entidad, en diciembre de 2008, es otro excelente avance en el proceso de integración regional en materia de defensa nacional.

En este punto se pueden destacar tres iniciativas: a) construcción de una visión geopolítica regional, b) formación en materia de defensa y c) ejercicios y unidades combinadas.

En primer lugar, durante el I Foro Suramericano de Políticas y Estrategias de Defensa de la Región se destacó la necesidad de coadyuvar en el desarrollo de una visión geopolítica regional. Asimismo, en el marco del IV Seminario Suramericano de Enfoques Conceptuales, Riesgos y Amenazas a la Región, se acordaron conceptos comunes y se propició la creación de un grupo de trabajo, a los efectos de cumplir con el punto 16 
de la Declaración de Paramaribo: "elaborar y presentar una propuesta de lineamientos estratégicos de UNASUR para la construcción progresiva y flexible de una visión compartida de defensa regional, la cual deberá exponerse en la próxima Reunión Ordinaria de este Consejo”. Durante los años 2014 y 2015, dicho Grupo de Trabajo continuó trabajando a partir de la propuesta, que se detalla a continuación, aprobada en el IV Seminario realizado en el año 2013:

- Respeto y fortalecimiento de los principios y objetivos establecidos en el Tratado Constitutivo de UNASUR y Estatuto del CDS.

- La cooperación y las medidas de fomento de la confianza mutua y seguridad como principales factores para la estabilidad y el fortalecimiento regional en el ámbito de la defensa.

- Fortalecer la capacidad de defensa de los Estados miembros como contribución a la estrategia de disuasión a nivel regional.

- La necesidad de coordinación y articulación entre las diversas instancias de UNASUR en lo referente a los temas de defensa.

- Buscar la autonomía regional en tecnologías sensibles para la defensa.

- Promover la responsabilidad y participación ciudadana en los temas de la defensa regional.

- Considerar la relevancia del uso soberano, la defensa y la protección de los recursos naturales como elemento para la construcción de la visión estratégica suramericana.

- Fortalecer la seguridad de la infraestructura y de las tecnologías de la información y comunicación en el ámbito regional.

En materia de formación el avance también fue significativo. Tanto Brasil como Argentina pusieron en marcha programas de formación en materia de defensa a través de los cuales funcionarios de las Ministerios de Defensa y de las Fuerzas Armadas de los países suramericanos podían compartir experiencias y debatir sobre la realidad en la materia en sus países y en la región. Particularmente en el caso Argentina, a partir del año 2012 y hasta el 2015 se realizó el Curso Suramericano de Formación en Defensa bajo la modalidad virtual y presencial en Buenos Aires. Este curso buscaba contribuir a "la formación, especialización y actualización de civiles en todo lo relativo al diseño, planificación, ejecución y supervisión de la política pública del sector defensa, a través de la puesta a disposición de conocimientos y herramientas teórico-conceptuales, jurídicas y de gestión" (SSAID 2013, 24).

La Escuela Suramericana de Defensa (ESUDE) fue propuesta durante la IV Reunión Ordinaria del Consejo de Defensa Suramericano (CDS), 
realizada el 28 de noviembre de 2012 en Perú. El proyecto ingreso al Plan de Acción 2013, durante el cual los países responsables, Argentina, Brasil y Ecuador, consensuaron la propuesta académica de la ESUDE, siendo seleccionada más tarde la República de Ecuador como sede. En tal sentido, se acordaron tres ejes de estudio:

a) Integración Regional y Cooperación Internacional en materia de defensa;

b)Defensa y Seguridad Regional;

c) Desarrollo y Participación Ciudadana en materia de defensa.

Se aprobó que la oferta académica inicial estuviera integrada por el Curso Avanzado en Defensa Regional CAD-SUL (Brasil) y el Curso Suramericano de Formación de Civiles en Defensa (Argentina). De este modo, los países de la UNASUR contarán con un ámbito de formación superior permanente de civiles y militares bajo una perspectiva de cooperación, integración y cultura para la paz, anclada en una concepción geopolítica sudamericana que se nutrirá de la interacción directa con el Centro de Estudios Estratégicos de Defensa del CDS (CEED). Esta tarea contribuirá a la construcción de una identidad estratégica estrictamente regional y profundizará la confianza mutua y la zona de paz sudamericana.

Finalmente, el Centro de Estudios Estratégicos para la Defensa (CEED) fue puesto en marcha el 26 de mayo de 2011. De esta manera, los doce países integrantes han dado un paso fundamental para iniciar un debate que lleve a la adopción de una visión estratégica común. En dicho Centro se efectuaron, entre otros, los siguientes trabajos:

Términos de referencia acerca de los conceptos de Defensa y Seguridad.

Informe sobre Crimen Organizado Transnacional y otras Nuevas Amenazas a la Seguridad Regional, en el cual se recomendó la creación de una instancia institucional para tratar estos temas y que, posteriormente, se materializó con la puesta en marcha del Consejo Suramericano de Seguridad Pública y Lucha contra el Delito Organizado.

Registro Suramericano de Gastos en Defensa 2006-2010 y que continúa actualizándose anualmente.

En el ámbito operacional cabe destacar la realización del ejercicio combinado regional sobre operaciones de mantenimiento de la paz, el cual tiene por objetivo "promover estándares de interoperabilidad combinada en materia de planeamiento y conducción de las operaciones de paz entre los Instrumentos Militares de nuestras naciones suramericanas" (SSAID 2013, 24). Asimismo, la creación de unidades combinadas binacionales en el ámbito suramericano "promueve y consolida mayores niveles de confianza 
mutua e incrementa los niveles de interoperabilidad en materia de acción conjunta y combinada con los países de la región” (MINDEF 2015, 229). En este aspecto fueron creadas en el año 2006 y 2008 la Fuerza de Paz Conjunta Combinada "Cruz del Sur" entre Argentina y Chile y la Fuerza de Paz Combinada "Libertador General San Martín" entre Argentina y Perú, respectivamente. La primera tiene por objetivo ser empleada como fuerza Stand By de la Organización de Naciones Unidas y cuenta con distintos niveles de conducción, desde los Ministerios de Defensa y Relaciones Exteriores hasta el que corresponde al estratégico operacional conformado por un Estado Mayor binacional con sede rotativa entre Buenos Aires y Santiago de Chile.

El segundo tiene por objetivo mejorar la infraestructura de los países afectados por conflictos internos y que está a disposición también de la Organización de Naciones Unidas. Cuenta con cuatro secciones: "1) sección de servicios: se encarga de la construcción, mejoramiento y reparación de infraestructura e instalaciones, 2) sección horizontal: su función está vinculada a todo lo referente a construcción y reparación de infraestructura vial, 3) sección vertical: tiene a su cargo la construcción y reparación de edificaciones y 4) sección agua: tiene la responsabilidad de obtener, potabilizar y distribuir agua" (MINDEF 2015, 230).

\section{CONCLUSIONES}

La Argentina se debe un debate profundo y el cambio de gobierno debería ofrecer un escenario inigualable. Si el presupuesto sin planeamiento es una dilapidación de recursos, el planeamiento sin recursos es un mero ejercicio intelectual (Pesce et al. 1999). En este sentido, si bien durante la gestión de Nilda Garré (2005-2010) en el Ministerio de Defensa se completó el entramado normativo que había quedado incompleto desde los años 1980 y se implementó el mismo, éste no se materializó en recursos presupuestarios. Ya desde principios del año 2009, con la renuncia de Germán Montenegro y su reemplazo por Gustavo Sibilla no se ejerció el control ministerial sobre el subordinado planeamiento estratégico militar. La llegada al Ministerio de Defensa de Arturo Puricelli, como claramente señala Anzelini et al (2014), se registraron deficiencias en el gobierno civil de la defensa, a lo que se sumó la ausencia de asignación presupuestaria. Durante la gestión de Agustín Rossi (20132015) en dicha jurisdicción, la falta de recursos y la omnipresencia del jefe del Ejército Tte. Gral. César Milani, bloquearon la implementación del PLANCAMIL, que el ministro intentó paliar con el lanzamiento del Segundo Ciclo de Planeamiento de la Defensa Nacional a fin de efectuar 
las correcciones presupuestarias necesarias en el PLANCAMIL 2011. Este proceso fue abortado en enero de 2016.

La política exterior y la política de defensa se encuentran íntimamente relacionadas en al menos dos sentidos. En primer lugar, la primera precede analíticamente a la segunda y, en este sentido, fija la orientación de la política de defensa. En este punto, Argentina suprimió las hipótesis de conflicto con los países de la región, lo cual impuso la adopción del planeamiento por capacidades militares. Pero este tipo de planeamiento en un contexto de incertidumbre y que requiere el rediseño del Instrumento Militar en el mediano plazo, trae aparejado la presencia de diversos tipos de riesgos para la seguridad estratégica del país. La mitigación de esos riesgos corresponde a la política exterior, particularmente, a la dimensión internacional de la política de defensa.

Esto no supone afirmar que la política exterior por sí solo pueda suplir el riesgo que supone reconstruir un Instrumento Militar y una política de defensa que no han formado parte de la agenda pública y de la agenda de gobierno desde 1983 hasta la fecha, salvo durante las gestiones de Raúl Borrás y Nilda Garré (Eissa 2015). Por el contrario, la política exterior contribuye a disminuir los riesgos que puedan devenir del escenario estratégico. Pero asimismo cómo la política exterior resulta esencial para el planeamiento por capacidades, la política de defensa también se relaciona a la política exterior, al menos de tres maneras. La virtuosa es cuando la política exterior "ilumina”, fija los lineamientos de la política de defensa. La segunda, la "viciosa”, es cuando la política de defensa se autonomiza de la política exterior. La realista es porque una política exterior sin política de defensa carece de dientes.

Durante la presidencia de Cristina Kirchner esa correlación e imbricación entre la política de defensa y la política exterior se materializó a través de la dimensión internacional de la primera que permitió acompañar las deficiencias en la implementación del PLANCAMIL 2011 que hemos señalado más arriba. Las acciones de una tuvieron su impacto en la otra. De la misma manera que la política exterior fijo la orientación de la política de defensa, las acciones de la primera, en esta agenda, acompañó el diseño de la segunda. Esto no necesariamente siempre es así. En nuestra historia, el desacople entre ambas fue lo más usual, siendo el ejemplo más doloroso la Guerra de Malvinas (1982).

Una no es posible sin la otra y su desunión ha sido uno de los problemas que ha enfrentado la inserción internacional de nuestro país. Ambas deben ir de la mano, caso contrario las fisuras en nuestro frente exterior debilita las posibilidades de contribución de estas políticas al desarrollo nacional. 


\section{BIBLIOGRAFÍA}

Alonso, Rodolfo, Guillermo Delamer, Federico Frischknecht, Mario Lanzarini, and Eulogio Moya. 1998. Estrategia. Teoría y práctica. Buenos Aires: Instituto de Publicaciones Navales.

Arteaga, F., and Fojón Lagoa, E. 2007. El planeamiento de la política de defensa y seguridad en España. Madrid: Instituto Universitario General Gutiérrez Mellado de Investigación sobre la Paz, la Seguridad y la Defensa.

Alconada Sempé, Raúl. 1996. “Democracia y política exterior”. In: Jalabe, Silvia Ruth (comp.). La política exterior argentina y sus protagonistas (1880-1995). Buenos Aires: GEL, 345-355.

Anzelini, Luciano, and Iván Poczynok. 2014. "El planeamiento estratégico militar en la Argentina (2003-2013): reflexiones en torno al gobierno político de la defensa”. Revista Brasileira de Estratégia E̊ Relações Internacionais 3, no. 6, 143-167. Porto Alegre: Núcleo Brasileiro de Estratégia e Relações Internacionais (NERINT) e ao Programa de Pós-Graduação em Estudos Estratégicos Internacionais (PPGEEI) da Faculdade de Ciências Econômicas (FCE) da Universidade Federal do Rio Grande do Sul (UFRGS).

Battaglino, Jorge. 2010. "La política militar de Alfonsín: la implementación del control civil en un contexto desfavorable”. In: R. Gargarella; Murillo M.; and Pecheny M. (comp.). Discutir Alfonsín. Buenos Aires: Siglo XXI, 161-184.

Colacrai, Myriam. 2004. "La política exterior argentina hacia los vecinos durante los '90. Documento de Trabajo no. 1. Buenos Aires: Universidad del CEMA.

Davis, Paul. 2002. Analytic architecture for capabilities-based planning, mission sistema analysis, and transformation. Washigton DC: National Defense Research Institute.

De Jomini, A. 1991. Compendio del arte de la guerra. Madrid: Ministerio de Defensa del Reino de España.

Entrevista reservada a Fuente X, 15 de enero de 2016.

Eissa, Sergio. 2005. Hielos Continentales. Las variables domésticas en la política exterior argentina. Buenos Aires: Fundación Síntesis.

2010. "Ni constante ni tan inconstante. Política exterior argentina en democracia”. Centro Argentino de Estudios Internacionales (CAEI). http://www.caei. com.ar/es/programas/pea/21.pdf. 
2013. "Redefiniendo la defensa: posicionamiento estratégico defensivo regional". Revista SAAP 1, no. 7, 41-64, Buenos Aires: Sociedad Argentina de Análisis Político.

2015. ¿La irrelevancia de los Estados Unidos? La política de defensa argentina (1983-2010). Buenos Aires: Arte y Parte.

. 2016. “¿Quo vadis defensa nacional?” El Economista (Sept.). Buenos Aires. http://www.eleconomista.com.ar/2016-09-quo-vadis-defensa-nacional/ .

2016. "Defensa Nacional: salir del Siglo XX". Clarín (Sept.). Buenos Aires.

Eissa, Sergio, Sol Gastaldi, and María Belén Canto. 2015. "Postura estratégica y planeamiento para la defensa en la República Argentina. Un aporte para el debate". Series Documentos de Trabajo No 31 . Buenos Aires: FADENA - Universidad de la Defensa Nacional.

Eissa, Sergio, and Jorge Souto. 2016. "Una aventura peligrosa”. El Economista (Oct.). Buenos Aires. http://www.eleconomista.com.ar/2016-10-una-aventura-peligrosa/.

Fontana, Andrés. 2006. "Política exterior argentina 1983 - 2005: visiones y cursos de acción”. In: Rodríguez Larreta, Horacio, and Sergio Berensztein (Eds.). Agenda para el desarrollo equitativo y sustentable. Buenos Aires: Editorial Temas. http:// pjgrupomayo.com.ar/documentos/descargas/11_1983_2005.pdf.

Fraga, Rosendo. 1998. "El concepto de las hipótesis de conflicto". In: Cisneros, Andrés (comp.). Política exterior argentina 1989-1999. Historia de un éxito. Buenos Aires: Nuevo Hacer. Grupo Editor Latinoamericano, 237-273.

Mendicoa, G. 2003. Sobre tesis y tesistas. Lecciones de enseñanza-aprendizaje. Buenos Aires: Espacio Editorial.

Pesce, Miguel, Eduardo Estévez, Sergio Eissa, and Analía Minteguiaga. 1999. El gasto en defensa del gobierno nacional (1993-1999). Buenos Aires: Fundación Karakachoff.

Pion-Berlin, David. 2001. "Civil-Military Circumvention. How argentine state institutions compesate for a weakened chain of command?" In: Pion-Berlin, David (ed.). Civil-military relations in Latin America. New Analytical Perspectives. North Caroline: The University of North Carolina Press, 135-160.

Russell, Roberto. 1990. "Política exterior y toma de decisiones en América Latina: aspectos comparativos y consideraciones teóricas”. In: Russell, Roberto (comp.). 
Política exterior y toma de decisiones en América Latina. Buenos Aires: Grupo Editor Latinoamericano, 255-274.

Russell, Roberto, and Juan Gabriel Tokatlian. 2003. El lugar de Brasil en la política exterior argentina. Buenos Aires: Fondo de Cultura Económica.

SSAID (2013), "Política Internacional de la Defensa Argentina-Gestión 2007-2013". Buenos Aires: Ministerio de Defensa - Subsecretaría de Asuntos Internacionales de la Defensa (SSAID).

Saín, M. 2010. Los votos y las botas. Estudios sobre la defensa nacional y las relaciones civil-militares en la democracia argentina. Buenos Aires: Prometeo.

Saín, Marcelo. 2000. “Quince años de legislación democrática sobre temas militares y de defensa (1983-1998)”. Desarrollo Económico. Revista de Ciencias Sociales 40, no. 157, 121-142. Buenos Aires: IDES.

Saín, Marcelo. 2003. "Nuevos horizontes, nuevos problemas. Las Fuerzas Armadas argentinas frente a las ‘Nuevas Amenazas` (1990-2001)”. In: López, Ernesto, and Marcelo Saín (comp.). Nuevas Amenazas. Dimensiones y perspectivas. Dilemas y desafíos para la Argentina y Brasil. Quilmes: Universidad Nacional de Quilmes, 173-220.

Taylor, S., and R. Bogdan. 1984. Introducción a los métodos cualitativos de investigación. La búsqueda de significado. Buenos Aires: Paidós.

Tello, Ángel, and Julio Spota. 2015. "Discusiones en torno al rol asignado a la inteligencia militar en la Argentina contemporánea”. Revista de Relaciones Internacionales, Estrategia y Seguridad 10, no. 2, 21-41. Bogotá: Universidad Militar de Nueva Granada.

Torres, Antonio. Circa 2009. "Instrumento Militar argentino del futuro". Revista de la Defensa, no. 5, 54-67. Buenos Aires: Ministerio de Defensa de la República Argentina.

Legislación Argentina

Expediente MD $\mathrm{N}^{\circ} 13.802$ del 30 de marzo de 2012 "Plan de Capacidades Militares 2011".

Decreto $\mathrm{N}^{\circ}$ 1691/2006 "Directiva sobre Organización y funcionamiento de las Fuerzas Armadas".

Decreto N 1714/2009 “Directiva de Política de Defensa Nacional 2009”.

Decreto N ${ }^{\circ}$ 1729/2007 “Ciclo de Planeamiento de la Defensa Nacional”. 
Decreto No 2645/2014 "Directiva de Política de Defensa Nacional 2014".

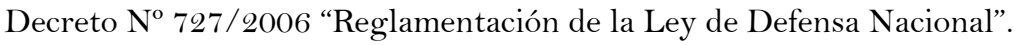

Ley No 23.554 de Defensa Nacional, 1988.

Ley No 24.059 de Seguridad Interior, 1992.

Ley $\mathrm{N}^{\circ} 24.429$ del Servicio Militar Voluntario, 1995.

Ley Nº 24.948 de Reestructuración de las Fuerzas Armadas, 1998.

Ley $\mathrm{N}^{\circ} 25.520$ de Inteligencia Nacional, 2001.

Ministerio de Defensa (Mindef) (2015) Libro Blanco de la Defensa 2015. Buenos Aires: Ministerio de Defensa de la República Argentina.

Resolución del Ministerio de Defensa No 414 del 27 de mayo de 2011 "Crea la Comisión para la elaboración del Plan de Capacidades Militares (PLANCAMIL)”.

Secretaría de Asuntos Internacionales para la Defensa (SAID) (2013) Política Internacional de la Defensa Argentina. Gestión 2007-2013. Buenos Aires: Secretaría de Asuntos Internacionales para la Defensa del Ministerio de Defensa de la República Argentina. 


\section{NOTAS}

1. Henri de Jomini (1991) fue uno de los primeros autores en usar este término.

2. Se parafrasea la definición de Marcelo Saín (2003: 234).

3. Integrantes de la Unión Cívica Radical fue fundada por Leandro Alem e Hipólito Yrigoyen entre otros, el 26 de junio de 1891. Es un partido liberal con una facción socialdemócrata. A lo largo de su historia se dividió en varias oportunidades, las principales en 1928, 1957, 2001 y 2007.

4. Integrantes del Partido Justicialista (conocido popularmente como peronista) fue fundado el 15 de enero de 1947 por Juan Domingo Perón. Se considera como "movimiento" y no como partido. Se pueden distinguir al menos tres corrientes ideológicas en su seno hasta el 2015: conservadurismo popular, socialcristianismo e izquierda nacional (no marxista).

5. Ver Eissa (2015). Una buena descripción de cómo se alcanzó el acuerdo es expuesta por Druetta (1989).

6. Para un mayor detalle de estos principios, se sugiere ver Eissa and Gastaldi (2014).

7. No se analizan todos los lineamientos. Para más detalles ver Eissa (2013; 2015).

8. Artículo $2^{\circ}$ Ley $\mathrm{N}^{\circ} 23.554$.

9. Artículo $1^{\circ}$ Decreto $\mathrm{N}^{\mathrm{o}} 727 / 2006$.

10. Artículo $1^{\circ}$ Decreto $\mathrm{N}^{\mathrm{o}} 727 / 2006$.

11. Artículo $2^{\circ}$ Ley $\mathrm{N}^{\mathrm{o}} 24.948$.

12. Decreto $\mathrm{N}^{\mathrm{o}} 1714 / 2009$.

13. Decreto $\mathrm{N}^{\mathrm{o}} 1691 / 2006$.

14. Decreto $\mathrm{N}^{\circ} 1691 / 2006$. Ver también el Decreto $\mathrm{N}^{\circ}$ 727/2006.

15. Para más precisiones sobre el planeamiento por capacidades, ver Eissa et al. (2015).

16. El planeamiento basado en capacidades fue originariamente empleado en forma aislada para el planeamiento nuclear durante los años 60, e incluso durante los años 70 para el posicionamiento de equipo militar estadounidense en el Golfo Pérsico. No obstante, se generalizó a partir del 2001 tras la aplicación de esta metodología en el planeamiento de la defensa estadounidense de la mano del Secretario de Defensa Donald Rumsfeld, aunque su primera mención data de la Revisión Cuadrienal de la Defensa de 1997 (Arteaga and Fojón Lagoa 2007). Este planeamiento pasó a reemplazar el modelo que había introducido el ex Secretario de Defensa Robert McNamara, basado en amenazas, y que se aplicó por al menos 40 años. 
17. Considerando el contexto estratégico, las diferentes metodologías de planeamiento de fuerzas pueden dividirse en dos categorías básicas: a) Planeamiento bajo certidumbre, que recoge el método de planeamiento basado en amenazas o por hipótesis de conflicto; b) Planeamiento bajo incertidumbre, categoría dentro de la cual podemos encontrar diversos métodos, tales como el basado en capacidades; el basado en escenarios; el método basado en efectos y; el método denominado enfoque global.

18. El nivel estratégico es el que corresponde a la conducción del conflicto y es propio de los estadistas. El nivel operacional es propio de los profesionales de la guerra y sus decisiones se traducen en planes. El nivel táctico corresponde al enfrentamiento de las fuerzas y resuelve problemas concretaos acotados en el tiempo y el espacio (Alonso et al., 1998).

19. El primer ciclo de planeamiento de defensa nacional debió haberse iniciado en el año 2007, pero el cambio de gobierno en ese año. Entrevista reservada a Fuente X, 15 de enero de 2016.

20. Tello y Spota (2015) disocian la política exterior de la política de defensa. Retomar el planeamiento por hipótesis de conflicto significa establecer un "enemigo" y salvo que los autores quieran dar marcha atrás con la política exterior argentina, vigente desde 1983, y volver a la hipótesis, vigente hasta la década del 1980 y que sobredimensionaría en la actualidad el Instrumento Militar, de conflicto a dos frentes con Chile y Brasil, A tal punto existió un desacople entre la política exterior y la política de defensa que el Reino Unido de Gran Bretaña nunca fue una hipótesis de conflicto del planeamiento militar y eso fue uno de los primeros errores que afectó la Batalla de Malvinas: lo que no se planifica en el Nivel Estratégico Nacional y Estratégico Militar no se corrige en el Nivel Estratégico Operacional. Finalmente, comenten tres errores conceptuales. El primero es que el planeamiento por capacidades no abreva en el idealismo wilsoniano. Tanto España como los Estados Unidos utilizan o han utilizado esta metodología de planeamiento, adaptándola a su realidad estratégica. Segundo, el artículo está sesgado más por el deseo y el pasado que por lo posible y el futuro. Revivir las hipótesis de conflicto con Brasil, Chile y el Reino Unido es anacrónico y violatorio de la Constitución Nacional y, por otro lado, adoptar las Nuevas Amenazas como hipótesis de empleo de las Fuerzas Armadas supone cabalmente su destrucción al convertirlas en policías militarizadas. Es lo que se denomina en otro trabajo como "realismo dependiente” y “realismo nostálgico" (Eissa 2016). Y finalmente, están y estamos analizando el caso argentino, no se puede extrapolar la realidad estratégica de México, Colombia y/o Brasil a la Argentina. Esto no necesita explicación. 
21. El primer signo de distensión con Brasil se remonta a la firma del Tratado de Corpus, Itaipú en 1979.

22. Ver también Russell and Tokatlian (2003, 43) y Fontana (2006).

23. Las motivaciones para avanzar en la relación con Brasil y Chile fueron distintas para Alfonsín y Menem. A pesar de ello, existe una fuerte continuidad. Ver Eissa (2010) y Eissa (2005).

24. Entrevista reservada a Fuente X, 15 de enero de 2016.

25. Entrevista reservada a Fuente X, 15 de enero de 2016. 


\section{LA RELACIÓN ENTRE LA POLÍTICA DE DEFENSA Y SU DIMENSIÓN INTERNACIONAL. EL CASO ARGENTINO (2011-2015)}

\section{RESUMEN}

El presente artículo sostiene que la postura estratégica defensiva y el planeamiento por capacidades adoptados, que implica la aceptación de riesgos políticos y estratégicos, requieren una política exterior activa y contribuyente a la defensa nacional. Para ello se estudia las políticas de defensa y exterior argentina entre 2011 y 2015.

Palabras claves: Argentina; Dimensión Internacional; Política de defensa; Planeamiento por Capacidades;

\section{ABSTRACT}

The present article argues that the strategic defensive posture and capacity planning adopted, involving the acceptance of political and strategic risks, require a foreign policy that is active and contributes to national defense. In order to study this relationship, Argentina's foreign and defense policy is analyzed between 2011 and 2015.

Keywords: Argentina; Defense Policy; International Dimension; Capability Based Planning. 